\title{
El castillo de Mula (1520-2020). Historia de la construcción de una fortaleza renacentista
}

The castle of Mula (1520-2020). History of the construction of a Renaissance fortress

\author{
José Antonio Zapata Parra
}

Ayuntamiento de Mula, Murcia, Spain, jzapata@aytomula.es

\begin{abstract}
Five hundred years after the construction of the castle of Mula, which was ordered to build the I Marqués de los Vélez, Pedro Fajardo Chacón, as a result of his expulsion from the town during the communal uprisings of the kingdom of Murcia. The fortress, a work of masonry, built on the old Andalusian citadel, has a novel construction in the southeast of the peninsular from the point of view of the multiaesthetic. The conservation of documentation related to its construction between 1520 and 1531, allows us to approach the work of the stonecutters and master gunners.
\end{abstract}

Keywords: Construction, fortress, military engineering, Renaissance.

\section{Introducción}

El 12 de septiembre de 1430 el rey Juan II concedía la villa de Mula a Alonso Yañez Fajardo II, adelantado mayor del reino de Murcia, por los leales y buenos servicios que le había prestado en la guerra contra el rey de Aragón. La donación proporcionaba el señorío con todos sus derechos: rentas, pechos, vasallos, justicia civil y criminal, mero y mixto imperio y facultad para establecer mayorazgo; pero con reserva real de tercias, alcabalas, monedas, pedido, minas y nombramiento de oficios concejiles, porque el concejo seguiría siendo de realengo.

Durante un siglo los diferentes señores de Mula mantuvieron esos privilegios, hasta que, a finales del siglo XV, don Pedro Fajardo Chacón, señor de Mula, adelantado mayor del Reino de Murcia y primer marqúes de los Vélez, comenzó su intromisión en el concejo muleño.

Esa intrusión señorial comenzó en 1495 con el nombramiento del doctor Fontes como alcalde

mayor de Mula, que empezó a inmiscuirse en la impartición de justicia, que hasta ese momento habían llevado los alcaldes ordinarios. Por otro lado, Pedro Fajardo comenzó a vender cargos concejiles, formando grupos de incondicionales a su causa. La hostilidad contra el marqués estalló en 1520, cuando los muleños, a principios de julio, se levantaron en comunidad, tomando la fortaleza y la villa, y expulsando a los hombres de los Fajardo. Para ello nombran nuevos alcaldes y ponen en la pared de la lonja esta frase: "hacerse lo que quisieramos"1. Unos meses antes, el 5 de mayo, el marqués ordenaba desde Cuevas de Almanzora que se activaran las obras del castillo (Fig. 1).

Tres semanas después, el 21 de julio, se presentaba el marqués de los Vélez frente a los muros de la villa. Con la mediación de los arcedianos de Lorca y Cartagena se llegó a un acuerdo con los vecinos para permitirle la entrada, no sin antes hacerle jurar ante un altar dispuesto en la 
puerta de Yechar (actual placeta del Pontarrón), dieciocho capítulos que no debía vulnerar. Los muleños reconocieron haberse levantado en armas, pero con el propósito de restablecer sus privilegios, usos y buenas costumbres, entre los que estaba que los oficiales del concejo elegirían a los nuevos miembros cada año, sin la intromisión del marqués.

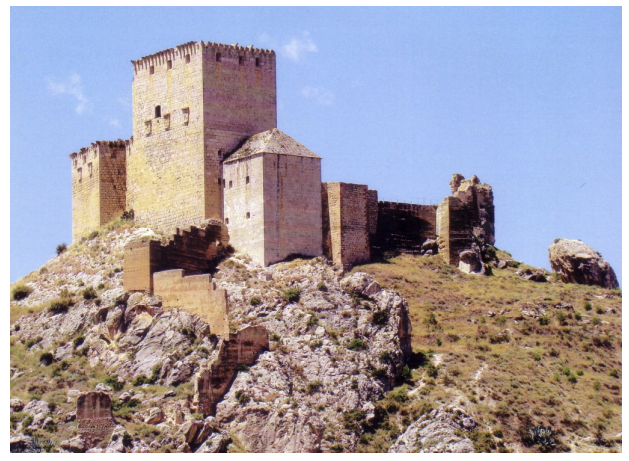

Fig. 1. El castillo de Mula visto desde el flanco oriental. Junto a él restos de estructuras de la alcazaba y de la muralla del albacar (J. Gutiérrez García).

Sin embargo, la humillación del marqués apenas duró cuatro años, pues don Pedro Fajardo logró un documento del rey Carlos I, en abril de 1524, en el que declaraba nulo el juramento firmado por la fuerza " $y$ en tienpo de tirania e por recobrar la dicha villa e fortaleza [...]"2. El 30 de junio, el gobernador y justicia mayor de Mula, don Rodrigo Fajardo, nombra nuevos cargos concejiles entre los partidarios del marqués.

En ese momento se encontraba en construcción la fortaleza, que comenzaba a tomar el aspecto que hoy día conocemos. Los muleños, sabedores de que el marqués contaba con el favor real, optaron por poner un pleito contra él en la Chancillería de Granada el 11 de febrero de 1525, argumentando, principalmente, entre otras demandas, que Mula era de la corona real y no de señorío.

\section{La construcción del castillo de Mula}

Según el historiador Nicolás Acero y Abad, las obras en el castillo se iniciaron hacia 1520, pues en el libro llamado Diferentes del Archivo Municipal pudo leer una carta del primer marqués en la que pedía activar la obra de la fortaleza. La carta fue remitida desde Cuevas de Almanzora con fecha de 5 de mayo de 1520. Dos meses después, se producía el levantamiento comunero, que sería resuelto con la entrada del marqués en la villa, el 21 de julio, bajo el juramento de guardar los usos, tradiciones y costumbres de Mula. Este levantamiento provocó la salida del antiguo alcaide, Hernán Gascón, de la fortaleza y la llegada de Luis Fajardo. Las obras bajo la dirección del nuevo alcaide debieron retomarse hacia 1522, según se desprende de las cartas enviadas al marqués, donde encontramos información sobre el gasto de estas entre enero y diciembre de 1523. Asimismo, en otra carta fechada en marzo de 1524, comunica al marqués que desde abril de 1523 tiene treinta hombres a su cargo y que el dinero para su manutención es escaso. Por lo tanto, las obras debieron de comenzar antes de 1523, año en el que estarían a pleno rendimiento, con el objetivo de que no se volviera a tomar la fortificación y quedara patente quién era el dueño y señor de la villa. El resultado fue la construcción de una nueva fortaleza, que tan sólo reaprovechó algunas estructuras precedentes (Fig. 2).

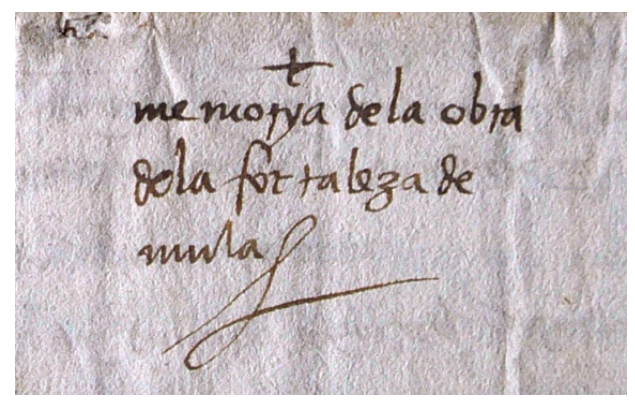

Fig. 2. Memoria de la obra de la fortaleza de Mula enviada por Luis Fajardo al marqués de los Vélez en 1524 (J.A. Zapata Parra).

En marzo de 1524, Luis Fajardo escribe al marqués sobre el estado de las obras en la fortaleza de Mula. El 10 de diciembre del año anterior, recién llegado de Málaga, donde había estado para resolver asuntos relacionados con su familia y su hacienda, se encontró muy avanzada, en la parte oeste, la torre triangular, a la que denomina "esquina de la proa como nao", o "torre puntada", 
pero que al comienzo de los fríos y las heladas se paró el asiento de sillares:

"Despues que vyne de Malaga que fue a X de dyzyembre halle hecho en la obra el esquina de la proa como nao et avya subido el esquina XII hyladas y no pasava la pared más a la otra buelta del esquina syno que en la misma esquina con las pyedras del esquina quedaba el travazon para la otra buelta y como se començasen los fryos y ela se paró la obra de asentar hasta primero de hebrero"3 (Fig. 3).

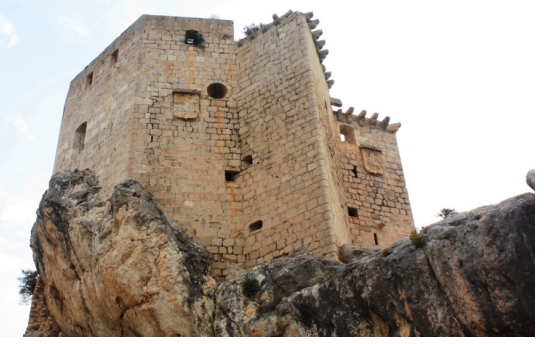

Fig. 3. Baluarte triangular en el extremo occidental de la fortaleza. Su forma la asemeja a la proa de un barco (J.A. Zapata Parra).

Le cuenta al marqués que, durante los meses que estuvo parada la obra, los trabajadores se ocuparon de limpiar la pedrera, que se había colmatado a causa del derribo de una de las torres de la fortaleza bajomedieval, posiblemente de mampostería, y que era conocida con el nombre de "la veleta", y que los maestros canteros estuvieron labrando los sillares que formarían las esquinas y cimientos, así como sus asientos:

"[...] la gente se ocupó en todo este tyempo de alympiar la pedrera porque estava muy ciega a causa de derrocar de la torre de la veleta en que se ocuparon algunos dyas y los maestros labraron esquinas y hazeras [...]". ${ }^{4}$

Le detalla pormenores como que las tempestades y fríos habían provocado que la garrucha utilizada para subir los materiales se cayera, y que la arena se hubiera acabado por el ritmo frenético de la obra. Asimismo, le dice que está buscando un nuevo picapedrero, porque al que contrató en Málaga lo habían matado:
“[...] no se ha hecho más en este mes de hebrero porque los dyas postreros hyzo tanta tempestad de ayres que nos desvarato el artefycio y helo [...], y la otra gente con la prysa que se ha dado en esta obra se a acabado el arena que ya no la hay adonde la avya [...] Para que aya más despacho en la obra e buscado otro picapedrero que el que avya de venir de Malaga que tenya concertado para esta obra mataronlo asy que yo busco otro". 5

Excusa el retraso de la obra por estar trabajando en las troneras de la fortaleza y, por lo tanto, los maestros canteros tardaban más en labrar cada sillar; describe incluso una de las troneras cercana a la puerta de "la albacara", que es la puerta que da a mediodía, donde se encontraba el albacar andalusí. Es interesante esta apreciación, ya que nos indica la altura a la que se encontraba la obra a comienzos de 1524 :

"Alguna tardanza ay en la obra a causa de hazer ya tantas bueltas y esquinas y labrar las troneras [...] A vuestra señoria escryby como avya asentado el tyro antochado en una tronera que haze traves a la puerta del albacara". ${ }^{6}$

Describe el aljibe, diciéndole al marqués que es una obra de baja calidad y debe ser reforzada, aunque él hubiera preferido demolerlo si no fuera por la premura de la obra. Este dato confirma que hay unas estructuras anteriores que están siendo reutilizadas y reformadas:

"Sabra vuestra ylustre señoria como el aljibe, esta obra tan falsa e tan vellaqua, que no se quien la mando hazer ni hizo tal porque esta de esta manera... Lo que se haze en el aljibe es que, apuntalando sobre madera en lo firme de la peña, apuradas aquellas granças, se rypia de buenas solturas para que se encorpore con lo rrypiado del adarve nuevo por que subydo de aquel asiento tan malo todas las otras paredes del aljibe son de buena argamasa" ${ }^{7}$

A la par que las reparaciones en el aljibe, la obra avanzaba en otras partes de la fortaleza, que identificamos gracias a las descripciones pormenorizadas de Luis Fajardo. En el cuerpo de guardia se están levantando dos grandes muros de cantería y eliminando parte de los antiguos, por encontrarse sueltos y en mal estado. Quiere un 
edificio resistente y perfecto, para que nunca lo puedan acusar de levantar una obra falsa. Apunta un dato muy interesante: que esta parte se adosará a la torre del homenaje a finales del mes de febrero, lo que nos indica que estaba ya avanzada. Con respecto a la torre del homenaje, se desprende de la carta que el maestro de obras estaba preocupado por el "solapo" que se estaba obrando en la torre, es decir, por la construcción del muro perimetral de ésta, que se asentaba justo en el borde de una gran peña rocosa con grandes grietas (Fig. 4):

"Ha se hallado esta peña con grandes fendas (grietas) y está muy carpyda del sol en lo alto y descubierto por donde no yba el adarve vyejo. Todo aquello yo lo hago pugnar y lo movedyzo derocallo abaxo... Algo se quita a esta causa el solapo que haze por ally la peña, asy que como a vuestra señoria escrybo medyado este mes o fin de el se juntara esta obra con el homenaje. Yo ya escryvy a vuestra señoria que la otra vuelta del homenaje que vuelve a donde se haze un gran solapo la peña sobre do está asentado el omenaje, todo aquella peña tiene grandes rresquebrados de alto a baxa y grandes fendas [...]".

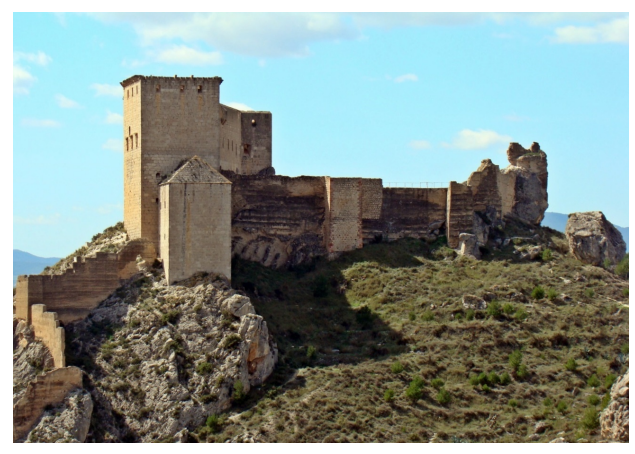

Fig. 4. Vista de los restos de la alcazaba musulmana con sus torres y lienzos (J. Gutiérrez García).

Otra de las zonas del castillo en la que está trabajando es la "torre nueva" localizada en el extremo occidental de la fortaleza, mirando a mediodía. En su interior se halla la sala de mando del cuerpo de guardia y bajo ella, un sótano. Luis Fajardo explica al marqués que es necesario hacer la bóveda que cubre el cuerpo de guardia y las salas de la torre por estas razones: para que toda la obra adquiera mayor resistencia, para que se creen nuevos espacios en la fortaleza, para que se puedan abrir las troneras de la plaza de armas y para que puedan igualarse los pavimentos del cuerpo de guardia, donde se encuentran las provisiones (Fig. 5).

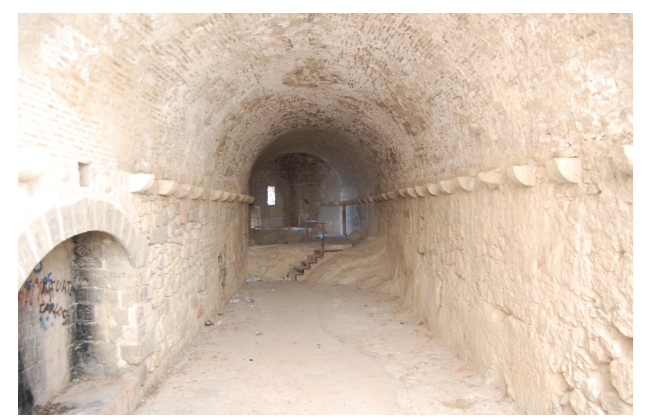

Fig. 5. Cuerpo de guardia del castillo de Mula (J.A. Zapata Parra).

También dice que va a construir la bóveda tomando como modelo la "bobeda vyeja", la cual es muy resistente por estar fabricada a base de mortero de cal y grandes lajas de piedra, y enlucida con yeso. Para sustentar la bóveda de la nueva torre levanta un gran muro de mampostería que separa el cuerpo de guardia de las demás estancias. Utiliza este muro para sostener la cimbra sobre la que fabricará la bóveda, con unas dimensiones de 3 varas de longitud. Esto hará que el castillo se eleve un "estado" por encima de la obra anterior, lo que supondrá una mejor defensa y colocación de la nueva artillería en la plaza de armas. En marzo de 1524, la torre tiene una altura de 27 hiladas, que equivalen a 9,174 m, y se termina antes del día de San Juan de ese mismo año, tiempo en el que comenzará la construcción del "omenaje nuevo", que identificamos con la tercera planta y la terraza de la torre del homenaje:

"Yo deseo, y tyene mucha necesydad esta obra a que se haga la bobeda en esta torre nueva por muchas causas, la principal por que se frange la obra junta y se encorpore en un cuerpo y masa y también porque aya espacio y anchura en la casa y poruqe se pueda hazer a peso y a razón con mejor medyda y conpas las troneras altas y porque se pueda ygualar el suelo en parejo del suelo de la bobeda en que esta agora los bastimientos [...] Y asimismo de que manera se debe hazer 
esta bobeda porque a mi me parece que se harya muy buena y muy recia de la masa y manera que esta la bobeda vyeja porque esta como un azero [...] Yo lo acabare para antes de San Juan y el omenaje nuevo se començara como se començo esta torre nueva que la primera piedra se asentó vyspera de San Juan. Por lo más alto de la torre nueva es de medyda que yo medy onze varas xxvii hyladas, esto está de alto". 9

Luis Fajardo informa al marqués de que en la zona que mira a Murcia hay una gran pendiente, por lo que es necesario forjar una cimentación de cuatro estados, eliminar una torre y parte de la muralla medieval por la que transcurre un adarve. La causa de estos derribos es la construcción de una puerta falsa o poterna en la parte donde se encuentran, y mantenerlas sería inconveniente para la seguridad y accesibilidad a la fortaleza. Otra de las causas que esgrime para eliminar la torre es su estado de conservación, pues a excepción de una esquina obrada en mampostería, posiblemente una reparación, el resto era de tapial que había perdido su cara exterior y estaba en proceso de desintegración. En la actualidad, se conservan parte del lienzo de la muralla y una torre más al sur, ambas con un adarve:

"Asimismo la dicha torrecilla no es de hazer caudal de ella a causa de estar muy mal labrada de mala obra. No tyene otra buena syno una esquina labrada de canterya. El argamasa que tyene es la cara de fuera y con esta de las aguas teñyda de pardyllo al parecer syn tocar en ella. Parece de las mas fyna argamasa que puede aver yo llegue a ella y la cate con un cochillo y toda se desahace como agua en cesto roto". ${ }^{10}$

La obra avanza a buen ritmo, pero a principios de febrero de 1524 se acaba el dinero asignado, por lo que Luis Fajardo le comunica al marqués que tuvo que adelantar de su bolsillo doscientos ducados para pagar a los trabajadores y para los demás gastos de la obra (Fig. 6). El administrador, un tal Juan Ramón, le había informado de que durante el año 1523 se gastaron 90000 maravedís que le había proporcionado el gobernador y alcalde mayor de Mula, don Rodrigo Fajardo:

"Y ya escryvy a vuestra señoria como no avya mas dyneros para la obra que ya eran gastados.
Yo quise saber lo que era gastado de Juan Ramón. Dyzeme que noventa mill maravedis desde enero de Mdxxiii [1523] años hasta fyn de diciembre de dicho año y que ya no tyene mas dyneros que no le dexo mas don Rrodrigo Fajardo. [...] Juan Ramón no tenya dyneros para pagar la gente y las otras cosas necesarias para esta obra. Yo deposyte en el doscientos ducados para que se gastasen por la orden que se paga y gasta la obra". ${ }^{11}$

Otro de los problemas con los que se encontrará es la falta de arena, que se había agotado de la rambla de donde la extraían. Como la del río era de baja calidad, se la compra a un vecino, que le suministra una arena de excelentes propiedades, con la que todos los maestros canteros están satisfechos:

“[...] con la prisa que se ha dado en esta obra se ha acabado el arena que ya no hay adonde la avya que como estava a boca de rambla acabose que ya no hay syno tasquin [...] En lo del arena yo dy un asiento con un vecino de aquí para que me de toda el arena que es menester para la dicha obra que no saldra a costa de vuestra señoria syno vyen por mas como por menos porque sy se cuenta lo que se dava a la gente cada dya de los dynerycos y los dyas que se ocupava en la hazienda vyene a con mucho menos de costa". ${ }^{12}$

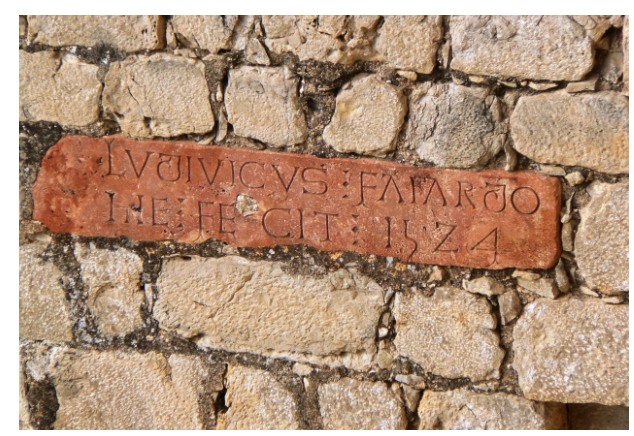

Fig. 6. Inscripción de Luis Fajardo en el castillo de Mula (J.A. Zapata Parra).

La fisonomía del castillo cambió definitivamente en el primer cuarto del siglo XVI, cuando el primer marqués comenzó una gran reforma para adaptar la fortaleza a los tiempos y a sus necesidades. Los muleños, acostumbrados desde época árabe a ver un castillo construido a base de ta- 
pial, comienzan a vislumbrar una obra de cantería. Así se refleja el cambio en una carta enviada por Carlos $\mathrm{V}$ al marqués de los Vélez el 16 de septiembre de 1524:

"[...] teneys una fortaleza en la dicha villa e que por algunas partes es de tapia e por otras de canteria e que agora nuevamente por partes de fuera diz que aveys fecho e hazeys una torre e un lienço de adarve que se junta con la dicha fortaleza e que por dentro hazeys otros nuevos rreparos de manera que donde hera muy flaca se haze muy recia e fuerte". ${ }^{13}$

En septiembre de 1525 continúan los problemas económicos en la obra, ya que no se envían las rentas de las villas de Molina, Librilla y Alhama, tal como se tenía establecido. Luis Fajardo no puede pagar a los oficiales y obreros contratados, mostrándose desesperado por no poder seguir los trabajos necesarios, llevando a cabo sólo los que puede, gracias a don Rodrigo Fajardo:

"Muy ilustre señor, esta obra estan grande y de tanta costa que cunple dalle gran recabdo. Y asy lo hazen estos a quienes mando vuestra señoria que me dyesen el rrecabdo de enviarme los dineros [...] Asy que hago saber a vuestra merced que de Molyna y de Lebrylla y de Alhama no me envían blanca. Suplyco a vuestra señoria que mande otro mandamiento con mayores penas que luego cobren todos los maravedis que se deven y me los envíen pues ya ha pasado el plazo". ${ }^{14}$

El 19 de septiembre de 1525 se habían asentado un total de 1.400 sillares, no pudiendo colocar más por falta de piedra. En ese momento hay un total de 14 obreros en el castillo, sin contar a los maestros canteros, trabajando en la labra de sillares, en la limpieza de la pedrera, produciendo cal y subiendo mampostería para el relleno de los muros. Es tal el trabajo, que es necesario construir una nueva garrucha para subir el material más pequeño. En ese momento se está trabajando en la "torre puntada", que es la terminada en forma triangular, conocida en la poliorcética con el nombre de esperonte, también en su trabazón con la torre nueva y el resto del cuerpo de guardia. Este acoplamiento le causa verdaderos quebraderos de cabeza, porque están adaptando estructuras viejas a la nueva planta. En cuanto a la altura de la obra, dice que tiene colocadas un total de trece hiladas:

"Yo tengo asentadas treze hyladas y otra hylada que agora asiento que son quatorze. No se a obrado mas a causa de aver faltaado pyedra labrada. Las pyedras labradas que estan asentadas de dentro y de fuera son por numero Mcccc [1400] pyeças de pyedra labrada [...] Tengo cynco picapedreros y los dos asentadores. Estos labran cada dya cada uno dos pyedras de las grandes y tres de las pequeñas. No se puede dar mas pryesa de la que se da y el almadanero para que de abasto traygo con el dos onbres que le ayudan y de esta manera da abasto de canterya para que labren. Y asimismo andan alympiando la pedrera otros cuatro onbres [...] Otro artificio armare esta semana mas del principal que sea mas sotyl, pues el grande basta para subyr la canterya grande. Asy que todo lo que se labrare se asentara y darse a pryesa que se pueda asentar cada quinze dyas una hylada [...] Se apareja la esquina que sale a la pedrera. Se haze agora en la peña su asiento. La obra por la parte de la torre puntada que sale a la villa, no llega la obra a emparejar con la torre, es la peña tan mala de labrar por quella parte, que bien hay que hazer que a todos nos tyene descalabrados toda la casa se rremude en ello". ${ }^{15}$

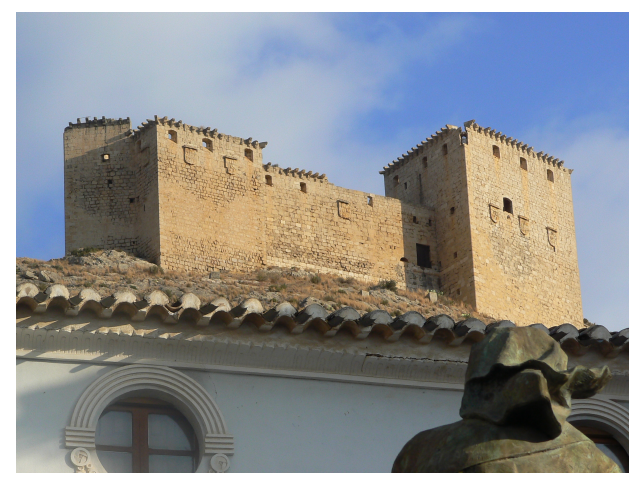

Fig. 7. El castillo visto desde la plaza del Ayuntamiento de Mula (J.A. Zapata Parra).

La última referencia a las obras en el castillo la hace el alcalde mayor de Mula, don Rodrigo Fajardo, en carta de 10 de octubre de 1525, en la que informa al marqués de que la obra va muy 
bien y que se ve desde la plaza y desde cualquier punto de la villa (Fig. 7):

“Aunque Loys Fajardo escribe a vuesa señoria que son quinse hiladas no son syno dyese seys por que despues que escrivio la carta asento otra y la obra va muy buena y de la plaça y de cualquier parte de la villa va muy bien". ${ }^{16}$

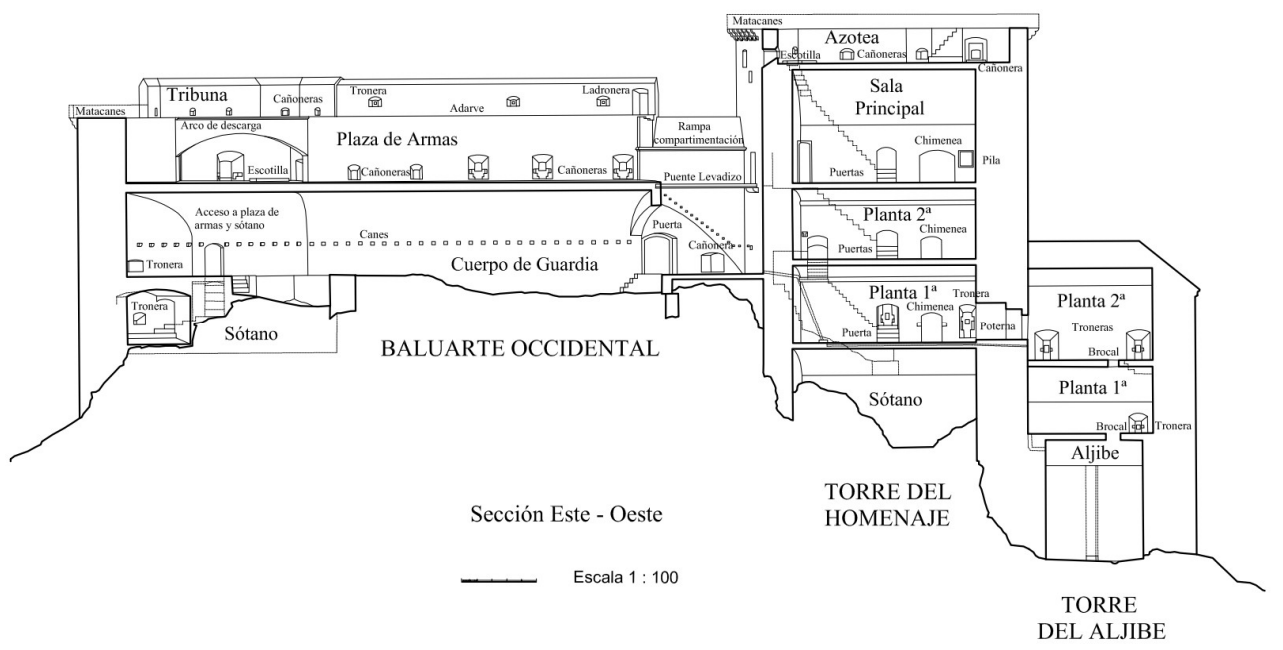

Fig. 8. Planimetria con las diferentes partes del castillo de Mula (J.A. Zapata Parra).

\section{Conclusiones}

¿En qué momento finalizó la construcción del castillo? Si atendemos a los datos que nos ofrecen las cartas de Luis Fajardo, en junio de 1524 se termina la llamada torre nueva, que identificamos con la torre en la que se inserta la inscripción "LVDIVICVS FAIARDO ME FECIT 1524" ("Luis Fajardo me hizo, 1524") (Fig. 6). En cuanto a la torre del homenaje, se encontraba en obras a la vez que la torre nueva y el aljibe, en el interior de una torre adosada a la del homenaje con una techumbre de piedra. Terminada la torre nueva, comenzó la obra nombrada "omenaje nuevo", que debe de corresponder a la planta tercera y terraza de la torre del homenaje, porque tienen una factura y técnica constructiva diferentes al resto. Sería terminada a finales de junio de 1525 , según se desprende de la memoria de la obra. Las últimas cartas conocidas, fechadas en septiembre y octubre de 1525, nos informan de que las obras en el castillo continúan, así como del número de hiladas colocadas en la fortaleza. Tan sólo hay una referencia a la "torre puntada", que en otra carta aparece cómo la "proa de una nao". Posiblemente, las obras que se están ejecutando corresponden a los adarves y muros del lado norte, donde se localiza la tribuna (Fig. 8).

No volvemos a tener información hasta marzo de 1531, fecha en la que sabemos que se está trabajando en la fortaleza, pues el maestro cantero Juan de Artiaga escribe una carta desde allí. Este maestro vizcaíno, posiblemente, fue contratado para rematar las obras, como sucedió en el castillo de Vélez Blanco, donde también fue contratado entre 1515 y $1517 .{ }^{17}$

Por lo tanto, podemos deducir que las obras que dieron forma al actual castillo de Mula se desarrollaron, aproximadamente, entre 1520 y 1531, según se desprende de los documentos hasta ahora conocidos.

\section{Notas}

${ }^{1}$ Franco Silva, 1995, p. 86, nota 73.

${ }^{2}$ Lemunier y González Castaño, 1989, p. 124. 
3-12 AGFCMS, Leg. 1145, carpetilla 1, doc. 32.

${ }^{13}$ AGS, RGS, diciembre de 1524. Cooper, 1991, vol. I.1, pp. 321-322.

${ }^{14}$ AGFCMS, Leg. 04. Cooper, 1991, vol. I.1, pp. 322-323.
${ }^{15}$ AGFCMS, leg.05. Cooper, 1991, vol. I.1, pp. 323-324.

${ }^{16}$ AGFCMS, leg. 04. Cooper, 1991, vol. I.1, pp. 37-38.

${ }^{17}$ Fernández Alcayna, 2001, pp. 27-34.

\section{Bibliography}

Acero y Abad, N. (1886). Historia de la muy noble y leal villa de Mula, Murcia.

Cooper, E. (1991). Castillos señoriales en la corona de Castilla, Consejería de Cultura y Turismo, 1991, Salamanca, 3 vols.

Fernández Alacayna, P. (2001). "Pleitos y enfrentamientos de la casa marquesal de los Vélez con los pobladores de su señorío y con la corona (s. XVI)", Revista Velezana, 20, pp. 27-34.

Franco Silva, A. (1995). El marquesado de los Vélez (siglos XIV- mediados XVI), Real Academia Alfonso X el Sabio, Murcia, pp. 39-41.

González Castaño, J. (1992). Una villa del Reino de Murcia en la Edad Moderna (Mula, 1500-1648), Real Academia Alfonso X el Sabio, pp. 212-213.

Gutierrez García, J. (2008). "Mula, cuna de los primeros Fajardo en el Reino de Murcia", Revista Velezana, 27, pp. 25-29.

Lemunier, G.; González Castaño, J. (1989). "Señores y oligarcas. Las luchas políticas en Mula durante los siglos XVI y XVII", Revista Áreas, 10, pp. 119-144.

Owen, R. (1980). Rebelión, monarquía y oligarquía murciana en la época de Carlos V, Universidad de Murcia, Murcia.

Rodríguez Llopis, M. (1998). Historia de la Región de Murcia, Murcia.

Rodríguez Pérez, R. (2012). "El II Marqués de los Vélez frente a su parentela", Revista Velezana, 30, pp. 6-19.

Roth, D. (2008). Vélez Blanco en el siglo XVI, Centro de Estudios Velezanos, Instituto de Estudios Almerienses, Vélez Rubio, 2 vol.

Ruiz García, A. (2002). El castillo de Vélez Blanco (Almería), Revista Velezana, Vélez Rubio.

Sánchez Maurandi, A. (1955). Historia de Mula, tomo I, Murcia.

Torres Fontes, J. (1953). Don Pedro Fajardo, Adelantado Mayor del Reino de Murcia, CSIC, Madrid.

Torres Fontes, J. (1978). “Los Fajardos en los siglos XIV y XV”, Miscelánea Medieval Murciana, IV, pp. 107-178.

Torres Fontes, J. (1998). "Bosquejo histórico de Mula en los siglos XIII y XIV”, Murgetana, 98, pp. 5-20.

Torres Fontes, J. (1999). "Bosquejo histórico de Mula en el siglo XV", Murgetana, 101, pp. 9-31.

Zapata Parra, J.A. (2015). El castillo de Mula (Murcia), Ayuntamiento de Mula y Fundación CajaMurcia, Mula. 